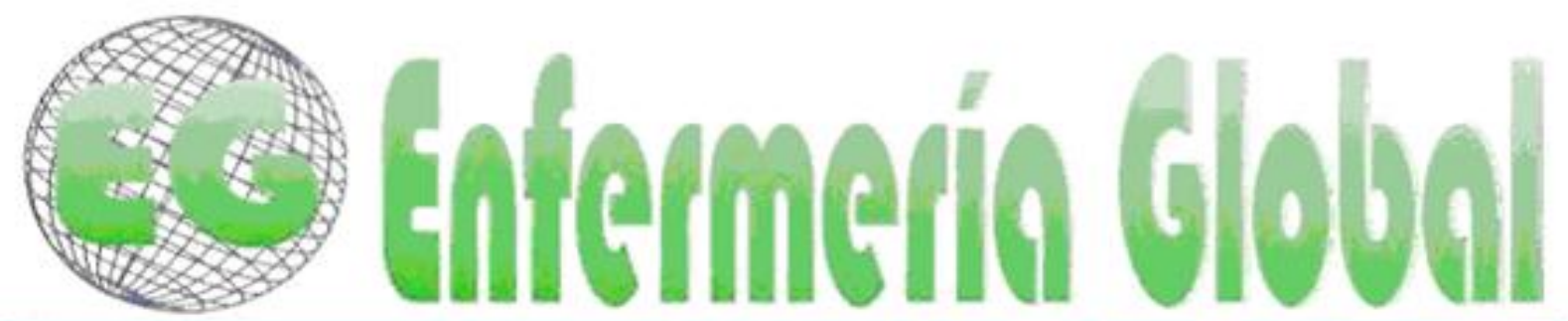

ISSN 1695-6141

Revista electrónica trimestral de Enfermería

No38

www.um.es/egloball

Abril 2015

\title{
CLÍNICA
}

\section{Evaluación del cumplimiento de un protocolo de prevención de Neumonía asociada a Ventilación mecánica en una UCI polivalente}

Assessment of compliance of a pneumonia prevention protocol associated mechanical ventilation in a polyvalent ICU

\section{*Villamón Nevot, María José}

*Enfermera. Hospital Obispo Polanco. Teruel. España. E-mail: mjvillamon@gmail.com

Palabras Clave: Neumonía asociada a ventilación mecánica; cuidados de enfermería; prevención; guías de práctica clínica.

Keywords: ventilator associated pneumonia mechanical; care nursing; prevention; Clinical practice guidelines.

\section{RESUMEN}

Objetivos: 1.Analizar el cumplimiento del protocolo mediante el registro del $80 \%$ de los cuidados de enfermería. 2. Describir la evolución de la densidad de incidencia de NAVM durante el periodo del estudio.

Metodología: Estudio descriptivo retrospectivo de los años 2009, 2010 y 2011. Se registró la realización de los cuidados incluidos en la prevención de NAVM en todos los pacientes en tratamiento con ventilación mecánica $(\mathrm{VM})>24$ horas, considerando que estaban cumplidos si el registro era $\geq$ $80 \%$. Se calculó el número de episodios NAVM por 1000 días de VM en el periodo del estudio.

Resultados: Se incluyeron un total de 94 pacientes. El cumplimiento de las medidas fue superior al $80 \%$ excepto en la valoración de la escala de Ramsay. El número de episodios de NAV por año fue 12,8 episodios por 1000 días de VM en 2008 y tras la implantación del protocolo fue de 4,57 en 2009, 6,83 en 2010 y 2,71 en 2011.

Conclusiones: 1. La frecuencia de registro de los cuidados ha sido buena. Para la mejora del registro de la valoración de la sedación, se hace necesaria la posibilidad de implantar un protocolo para el destete. 2. La incidencia de NAVM ha descendido tras la implantación del protocolo.

\section{ABSTRACT}

Aims: 1. Analyze compliance of $80 \%$ of the protocol. 2. Describe the evolution of the density of VAP during the study period. 
Methodology: Retrospective and descriptive study in years 2009, 2010 and 2011. They record the delivery of care including the prevention of VAP in all patients on mechanical ventilation (MV) $>24$ hours, considering they were met if the record was $\geq 80 \%$. We calculated the number of episodes of VAP per 1000 days of VM in the study period.

Results: A total of 94 patients were included. The performance of all measures was over $80 \%$ less except Ramsay assessment scale. The numbers of episodes of VAP per year were 12.8 for 1000 days of mechanical ventilation in 2008 and then implementation measures were 4.57 episodes in 2009, 6.83 episodes in 2010 and 2.71 episodes in 2011.

Conclusions: Frequency of care record has been good. To improve the record of the assessment of sedation, it is necessary the possibility of implementing a protocol for weaning. 2 . The incidence of VAP has declined after implementation of the protocol.

\section{INTRODUCCIÓN}

La ventilación mecánica es un tratamiento frecuente utilizado en $\mathrm{UCl}$ y se asocia a potenciales complicaciones, siendo la neumonía y la dependencia del ventilador dos de las más frecuentes y que más preocupan ${ }^{(1)}$.

La Neumonía asociada a ventilación mecánica (NAVM) se define como una infección del parénquima pulmonar que aparece en pacientes después de estar sometidos a 48 horas de ventilación mecánica, que no estaba en incubación ni presente en el momento de la intubación ${ }^{(2)}$ o que es diagnosticada en las 72 horas siguientes de la extubación y retirada de la ventilación mecánica ${ }^{(3,4)}$.

La NAVM se puede clasificar en temprana o tardía según se desarrolle en los primeros 4 días de la admisión del paciente o uso de la VM o a partir del quinto día ${ }^{(3-}$ 5).

El riesgo de desarrollar neumonía aumenta con el tiempo de ventilación invasiva, si este es menor de 24 horas, los pacientes tienen un riesgo 3 veces mayor de presentar neumonía que aquellos que no reciben este tratamiento, mientras que si este es superior a 24 horas el riesgo se incrementa entre 6 y 21 veces ${ }^{(6,7)}$.

La NAVM es la segunda causa de infección nosocomial ${ }^{(4)}$. Según los datos aportados por el proyecto Neumonía Zero en $2010^{(8)}$, la tasa de incidencia en 2010 se encontraba en torno a 12 episodios por 1000 días de ventilación mecánica. Estas cifras deben tenerse en cuenta ya que la NAV es la primera causa de mortalidad atribuible a infecciones nosocomiales y tiene una alto impacto sobre la morbimortalidad de los pacientes, los días de estancia en UCl y en los costes ${ }^{(4,7,8-11)}$. La mortalidad en pacientes con NAVM oscila entre $24-75 \%{ }^{(5,8)}$, el aumento de la estancia en $\mathrm{UCl}$ oscila entre 7 y 9 días ${ }^{(4,5,8)}$ y todo esto, generando un coste adicional entre 3.000-40.000 dólares en estudios publicados en $2005^{(4,8,10)}$ y en un estudio más reciente publicado en 2009 el coste calculado era entre 9000-31000 euros ${ }^{(5)}$.

Existen numerosos factores de riesgo que pueden llevar al desarrollo de la NAVM. Los no modificables son las características inherentes del paciente como la edad, la severidad de la enfermedad y otras enfermedades concominantes Los modificables son aquellos sobre los que si actuamos de una manera correcta podemos lograr que el riesgo disminuya como la higiene de manos, la educación sanitaria y la posición del paciente. Es por esto, por lo que nuestro objetivo deberá centrarse en realizar 
actividades sobre estos factores modificables que nos permiten disminuir el riesgo ${ }^{(4,}$ $5,11)$

Los cuidados para prevenir la NAVM se incluyeron por primera vez en el año 2006 en la campaña "The 100k lives campaign" observando una disminución del $59 \%$ de la tasa de NAVM en aquellas unidades que habían cumplido más del $95 \%$ de las medidas propuestas. En nuestro país ni la Sociedad Española de Medicina Intensiva, Crítica y Unidades Coronarias (SEMICYUC) ni la Sociedad Española de Enfermedades Infecciosas y Microbiología Clínica (SEIMC) habían propuesto guías ni recomendaciones y eran los expertos los que elegían medidas que a su juicio eran más efectivas, menos mórbidas y más fáciles de cumplir, pero no se había implantado un programa a nivel nacional ${ }^{(8)}$. Todos los paquetes de medidas incluyen medias farmacológicas y no farmacológicas y es, sobre estas últimas, donde la enfermería tiene un papel protagonista debido a su papel como responsable del cuidado del paciente. Todas estas medidas se encuentran descritas en diferentes guías clínicas según su nivel de evidencia científica y recomendación ${ }^{(7)}$

Las investigaciones publicadas sobre el uso de paquetes de prevención de NAVM son escasas y en muy pocos se engloba de forma conjunta un paquete de medidas. La elección de componentes se basa en la evidencia actual que aportan diferentes organismos como la clasificación GRADE, Task Force Canadiense, las recomendacines del Centers for Disease Control and Prevention (CDC) o las que realiza el Instituto Joanna Briggs y se ha demostrado que todos los paquetes de cuidados reducen la incidencia de NAVM. ${ }^{(3)}$

1. Formación del personal: resaltando la manipulación de vía aérea ${ }^{(5,9,12-15)}$. Concretamente el estudio "Evidence-Based Practice: Use of the Ventilator Bundle to Prevent Ventilator-Associated Pneumonia" asegura que una sesión educativa de 30 minutos del personal de enfermería mejora el conocimiento y el uso de estrategias de prevención.

2. Lavado de manos y guantes cuando se entre en contacto con secreciones y equipos de ventilación. La desinfección de manos con soluciones de base alcohólica ha aumentado el cumplimiento de esta medida del $48 \%$ al $66 \%$. $(2,3,5,8-13)$

3. Intubación orotraqueal mejor que la nasotraqueal ya que la segunda puede producir sinusitis y esta es un factor de riesgo de desarrollar NAVM $(1,2,3,5,6,9,10,12)$

4. Favorecer todos los procedimientos que permitan disminuir de forma segura la intubación y/o su duración, porque a mayor duración existe un mayor riesgo de NAVM ${ }^{(2,5,6,8,16,17)}$. El Institute for Health Improvement (IHI) de Massachussetes ha identificado la interrupción diaria de la sedación y la valoración diaria del destete como una de las 4 intervenciones claves para disminuir la incidencia de NAVM ${ }^{(1)}$.

5. Evitar la posición de decúbito supino. Se recomienda elevación de la cabecera de la cama entre $30-40^{\circ}$ (siempre que no exista contraindicación). A pesar de ser una medida fácil en un estudio observacional se detectó que solo el $85 \%$ de los pacientes mantenían la elevación a 45 ${ }^{(18)}$.Respecto a las camas cinéticas, aunque la medida es eficaz, no se puede recomendar por falta de datos sobre el riesgo-beneficio ${ }^{(3,5,6,9,10,19)}$ Esta medida es especialmente importante en pacientes que reciban nutrición enteral ya que se ha considerado un factor de riesgo por la posibilidad de aspiración del contenido gástrico ${ }^{(2,3,5,6,8-13,16,17)}$. De ahí la recomendación de realizar una comprobación del volumen gástrico 
residual cada 4-6 horas y aplicar retención de la alimentación 1 hora si el volumen es $>1-1,5$ veces la cantidad administrada $0150 \mathrm{ml}$ en bolo ${ }^{(15)}$.

6. Presión óptima de neumotaponamiento entre $20-30 \mathrm{~cm} \mathrm{H}_{2} \mathrm{O}$ con el objetivo de prevenir el paso de patógenos hacia el tracto inferior. Una presión mayor puede provocar una lesión de la tráquea ${ }^{(3,5,11,12,16)}$. En tres estudios ${ }^{(2,8,9)}$ se hace mención al número de determinaciones. Un control continuo dependerá de la disposición material del hospital por lo que se aconseja disponer de un sistema estandarizado con control de la presión cada 8 horas.

7. Las secreciones que se acumulan por encima del tubo endotraqueal es un factor de riesgo de NAVM, pero se desconoce la frecuencia de aspiración recomendada. Existe un estudio prospectivo que concluye que si el volumen es alto $(>10 \mathrm{ml})$ aspiración cl2 horas o más a menudo ${ }^{(20)}$. Respecto a los sistemas de drenajes de secreciones se ha demostrado que disminuye la incidencia de NAVM de comienzo precoz en pacientes con una duración de la ventilación mecánica > 72 horas, pero no hay disminución de la mortalidad, de la estancia en UCI ni de los días de ventilación mecánica ${ }^{(3,5,6,8,9,10,16-18,20)}$. En cuanto a la utilización de sistemas cerrados 0 abiertos no encuentran diferencias en relación a NAVM pero sí se habla de ventajas e inconvenientes como que el abierto está asociado a desaturación arterial e inestabilidad hemodinámica y el cerrado mayor colonización y es más caro ${ }^{(21-23)}$.

8. Humidificadores e intercambiadores de calor-humedad: todos los estudios coinciden en que no tienen relación con una disminución de la NAVM, pero que los intercambiadores de calor-humedad reducen la colonización bacteriana, la condensación y tienen un menor coste ${ }^{(2,3,5,9,11,16,24)}$. Se desaconseja el cambio rutinario de las tubuladuras porque no disminuye la NAVM y sí aumentan los costes a no ser que estén visiblemente sucios y con un nuevo paciente ${ }^{(2,5,6,8-}$ $10,12,16,25)$. Lo que no queda resuelto es la duración de las tubuladuras.

9. Se recomienda la desinfección con antiséptico como la clorhexidina $0.12-2 \%$ cada 8 horas ${ }^{(8-13,16,18,26)}$.

10. Descontaminación selectiva digestiva (DDS): se pretende prevenir el desarrollo de infecciones endógenas mediante la aplicación tópica en orofaringe y tubo digestivo de una mezcla de antimicrobianos no absorbibles. Es la medida con mayor número de ensayos $(n=60)$ y la única que ha demostrado su impacto contra la mortalidad por NAVM. La no utilización es debido a que la práctica es compleja, al miedo a resistencias y al alto coste por lo que su aplicación dependerá de la disponibilidad de la UCI ${ }^{(8-10,12,13,14,16,19,26)}$.

En la UCl del Hospital de Teruel, que es nuestro ámbito de estudio, se detectó una tasa de incidencia en 2008 de 12,5 episodios de NAVM por cada 1000 días de ventilación mecánica, y por este motivo, sus profesionales decidieron hacer una revisión bibliográfica para implantar medidas que disminuyeran la incidencia. Para registrar estas medidas, se elaboró un cuño que se impregnaba en las gráficas del paciente que estaba bajo tratamiento con ventilación mecánica.

En marzo del 2011, la UCI empieza a formar parte del proyecto Neumonía Zero liderado por la Agencia de Calidad del Ministerio de Sanidad y dirigido en su aplicación por la SEMICYUC. Este proyecto tiene como objetivo reducir la tasa media estatal de densidad de incidencia a 9 episodios de NAVM por 1000 días de VM y para ello propone un paquete de medidas de obligado cumplimiento y otras optativas altamente recomendadas, todas ellas basadas en los criterios de evidencia científica del GRADE (Grading of Recommendations Assessment, Development and Evaluation Working Group). Tabla I. Con la incorporación a este proyecto se introducen mejoras 
como el registro numérico de la presión del neumotaponamiento, el no cambio semanal de las tubuladuras del respirador y el nivel de sedación deseado. Además se proporciona al personal de enfermería la formación necesaria que hasta este momento no se había realizado ${ }^{(8)}$.

Tabla I.Medidas de cumplimiento para la prevención de NAVM del poyecto Neumonia Zero.

\begin{tabular}{|c|c|}
\hline Medidas de Obligado cumplimiento & Medidas Optativas altamente recomendadas \\
\hline $\begin{array}{l}\text { a) Formación y entrenamiento apropiado en la } \\
\text { manipulación de la vía aérea } \\
\text { b) Higiene estricta de las manos antes de } \\
\text { manipular la vía aérea } \\
\text { c) Higiene bucal utilizando clorhexidina } \\
\text { (0,12\%- } 0,2 \% \text { ) } \\
\text { d) Control y mantenimiento de la presión del } \\
\text { neumotaponamiento ( }>20 \mathrm{~cm} \text { H2O) } \\
\text { e) Evitar, siempre que sea posible, la posición } \\
\text { de decúbito supino a } 0 \text { o } \\
\text { f) Favorecer los procedimientos que permitan } \\
\text { disminuir de forma segura la intubación y/o su } \\
\text { duración } \\
\text { g) Evitar los cambios programados de las } \\
\text { tubuladuras, humidificadores y tubos } \\
\text { traqueales. }\end{array}$ & $\begin{array}{l}\text { a) Aspiración continua de secreciones } \\
\text { subglóticas } \\
\text { b) Descontaminación selectiva del tubo } \\
\text { digestivo (completa u orofaríngea) } \\
\text { c) Antibióticos sistémicos (dos días) durante la } \\
\text { intubación en pacientes con disminución del } \\
\text { nivel de consciencia }\end{array}$ \\
\hline
\end{tabular}

Los objetivos planteados en este estudio son:

1. Analizar el cumplimiento del protocolo de prevención de NAVM.

2. Describir la evolución de la densidad de incidencia durante el periodo del estudio.

\section{METODOLOGIA}

Se realiza un estudio descriptivo retrospectivo. El ámbito de estudio es la unidad de cuidados intensivos del Hospital Obispo Polanco de Teruel. Esta UCI es de carácter polivalente y tiene una capacidad de 6 camas. El ratio enfermera/paciente es 1/3.

La población a estudiar son pacientes ingresado en la unidad en los años 2009, 2010, 2011 portadores de IOT sometidos a ventilación mecánica invasiva > 24 horas. También se incluirán aquellos pacientes que habiendo estado bajo IOT se les realiza traqueotomía y siguen recibiendo ventilación mecánica invasiva.

Quedan excluidos los pacientes que reciben tratamiento con $\mathrm{VM}<24$ horas.

El tipo de muestreo será no probabilístico consecutivo. La muestra estará compuesta por todos los pacientes que han sido ingresados en la unidad y reciben tratamiento con ventilación mecánica durante los años 2009, 2010 y 2011.

Las variables a estudiar son:

a) Demográficas: Sexo, edad, diagnóstico de ingreso. 
b) Variables explicativas:

Puntación APACHE II al ingreso (en las primeras 24 horas) ${ }^{(A n e x o ~ 1) . ~}$

Cuidados: todos ellos registrados una vez por turno y con la variable dicotómica $\mathrm{SI} / \mathrm{NO}$

- higiene de manos por turno: utilizando solución alcohólica o jabonosa.

- posición semiincorporada de la cabecera de la cama por turno.

- higiene oral con clorhexidina por turno: se utiliza clorhexidina $0,12 \%$.

- monitorización de presión de globo endotraqueal por turno: mediante manómetros marca Portex. Se considera presión óptima $20-30^{\circ} \mathrm{cmH}_{2} \mathrm{O}$.

- aspiración subglótica continúa. valoración de la escala de Ramsay (anexo 2) por turno. Se realiza un corte a la semana para la valoración en el protocolo.

- comprobación de tolerancia de la nutrición enteral por turno: en todos los pacientes que reciben alimentación enteral se conecta la SNG a derivar a bolsa durante una hora en cada turno.

- utilización humidificador: se realiza por prescripción médica.

c) Variables resultado:

Episodios de NAV por 1000 días de ventilación mecánica.

Registro del protocolo.

Los datos han sido recogidos por la investigadora procedentes de historial médico de la $\mathrm{UCl}$ y los registros de los protocolos de cuidados a pacientes con VM, registro de sedación de paciente y registro de administración parenteral y enteral (Anexo3). Estos protocolos han sido rellenados por las enfermeras de la unidad encargadas de cada protocolo y los datos procedían de los registros de la gráfica de enfermería. (El cuidado no registrado se considera no realizado)

Los datos referentes a tasas de neumonía asociada a ventilación mecánica (NAVM) fueron recogidos por los internistas de la unidad. Los criterios de diagnóstico de NAVM fueron: diagnóstico clínico; clínica compatible más nuevo y persistente infiltrado radiológico, extensión de un infiltrado previo y empeoramiento clínico y diagnóstico etiológico rutinario mediante cultivo de aspirado bronquial.

\begin{tabular}{|c|c|c|c|c|c|c|c|c|c|c|c|}
\hline \multirow{2}{*}{$\begin{array}{l}\text { Figura 1: Cronograma } \\
\text { de la investigación }\end{array}$} & \multicolumn{3}{|c|}{2011} & \multicolumn{8}{|c|}{2012} \\
\hline & Oct & Nov & Dic & Ene & Feb & Marz & Abr & May & Jun & Jul & Agost \\
\hline \multicolumn{12}{|l|}{ Revisión bibliografica } \\
\hline Recogida de datos & \multicolumn{11}{|c|}{$\curvearrowright \sqrt{2}$} \\
\hline Análisis datos & \multicolumn{11}{|c|}{$\Rightarrow$} \\
\hline Conclusiones & \multicolumn{11}{|c|}{$\longrightarrow$} \\
\hline
\end{tabular}


Para la elaboración y el tratamiento estadístico de los datos se ha utilizado la base de datos "Excel" y el software "R Commander" versión 2.14.0.

Se ha utilizado estadística descriptiva: distribución de frecuencias para variables cualitativas y medias, desviación estándar, valor mínimo y máximo para variables cuantitativas.

Para la comparación de los datos se ha realizado la media de los porcentajes de los tres turnos para cada año y después se ha usado la prueba de T de Student.

El cálculo de la densidad de incidencia se realizó dividiendo el número de casos de NAVM en cada año del periodo de estudio, entre el número de días de ventilación mecánica invasiva de todos los pacientes que estuvieron con tratamiento de ventilación mecánica invasiva durante ese año y el resultado se multiplicó por 1000.

№ NAVM (año 2009,2010 2011)

№ de episodios NAVM por 1000 días de VM = $\mathrm{X} 1000$

Días VM (años 2009,2010,2011)

\section{Consideraciones éticas}

Se solicitó autorización para el acceso a las historias a la dirección médica del hospital.

No se solicitó consentimiento a los pacientes por tratarse de cuidados que están sistematizados dentro de los protocolos.

La memoria fue enviada para su aprobación al comité ético de la Universidad Jaume I.

\section{RESULTADOS}

Un total de 94 pacientes fueron incluidos en el estudio (35 en 2009, 40 en 2010 y 19 en 2011). De estos, 58 eran hombres $(61,7 \%)$ y 36 mujeres $(38,3 \%)$. La media de edad era 67,04 años con una desviación estándar (DE) de 16,18.

Respecto al diagnóstico de ingreso se agruparon en 3 categorías: 35 pacientes con patología médica $(37,23 \%), 33$ pacientes con patología quirúrgica $(35,11 \%)$ y 26 pacientes con patología respiratoria $(27,66 \%)$.

La puntación de APACHE II media fue de 18,36 (DE 9,06), no siendo registrada en el informe médico de 13 pacientes. 
Los pacientes estuvieron con ventilación mecánica una media de 11,04 días con DE 9,11 (min 2 días; máx 54 días).

De los 94 pacientes, 30 pacientes precisaron la utilización de humidificador, 35 llevaban aspiración de secreciones continua y en 26 pacientes fue necesario realizar traqueotomía.

Respecto a los cuidados registrados, los resultados obtenidos en cada año se muestran en la tabla $\mathrm{V}$.

Tabla V: Cuidados registrados por año y turno en los protocolos para el cuidado a los pacientes con ventilación mecánica.

\begin{tabular}{|c|c|c|c|c|c|c|c|c|c|c|c|c|c|c|c|c|c|c|}
\hline & \multicolumn{6}{|c|}{2009} & \multicolumn{6}{|c|}{2010} & \multicolumn{6}{|c|}{2011} \\
\hline & Mañana & (n) & Tarde & (n) & Noche & (n) & Mañana & (n) & Tarde & (n) & Noche & (n) & Mañana & (n) & Tarde & (n) & Noche & (n) \\
\hline Higiene oral & 99,05 & 318 & 89,62 & 321 & 83,75 & 323 & 97,3 & 371 & 93,87 & 385 & 93,4 & 379 & 99,58 & 237 & 93,31 & 239 & 89,54 & 239 \\
\hline Higiene manos & 95,3 & 234 & 89,36 & 235 & 83,05 & 236 & 97,57 & 371 & 95,47 & 375 & 95,78 & 379 & 98,31 & 237 & 91,63 & 239 & 90,79 & 239 \\
\hline Med globo & 91,72 & 318 & 81,23 & 321 & 74,04 & 313 & 96,23 & 371 & 90,67 & 375 & 89,18 & 379 & 97,05 & 237 & 91,21 & 239 & 90,79 & 239 \\
\hline Pos.cabecera cama & 97,21 & 215 & 89,35 & 216 & 81,94 & 216 & 96,5 & 371 & 95,73 & 375 & 94,46 & 379 & 100 & 237 & 92,8 & 239 & 91,21 & 239 \\
\hline N.Enteral & 80,55 & 118 & 65,83 & 118 & \begin{tabular}{|l|}
65,25 \\
\end{tabular} & 118 & 95,12 & 123 & 96,15 & 130 & 94,3 & 130 & 95,09 & 102 & 93,14 & 102 & 93,14 & 102 \\
\hline Ramsay & 54,17 & 33 & 54,17 & 36 & 48 & 37 & 59,6 & 54 & 50,94 & 52 & 57,4 & 54 & 63,16 & 19 & $\mid 63,16$ & 19 & 63,16 & 19 \\
\hline
\end{tabular}

Todos los cuidados, excepto la evaluación de la sedación mediante la escala de Ramsay, se cumplieron en más del $80 \%$ en todos los años.

Para el tratamiento estadístico de los datos tomamos como valor significativo $p<0,05$. Si comparamos la evolución de cada cuidado por año podemos observar que:

- $\quad$ No existe diferencia significativa entre los tres años en:

1. Higiene oral $(2009-2010 p=0.34 ; 2010-2011 ; p=0.72 ; \quad$ y 2009-2011 $p=$ $0.16)$.

2. Higiene de manos $(2009-2010 p=0.14 ; 2010-2011 p=0.26 ; 2009-2011 p=$ 0.12 ).

3. Medición del globo de neumotaponamiento (2009-2010 $p=0.08 ; 2010-2011$ $\mathrm{p}=0.09$; 2009-2011 $\mathrm{p}=0.08$ ).

4. Posición de la cama $(2009-2010 p=0.25 ; 2010-2011 p=0.72 ; 2009-2011 p$ $=0.12)$.

- Existe diferencia significativa:

1. Comprobación del débito de la nutrición enteral: existe una diferencia significativa entre los años 2009-2010 ( $p=0.03$ ) y 2009-2011 ( $p=0.03$ ). 
2. Escala de valoración de Ramsay: a pesar de que es el único cuidado que no se cumple en el protocolo, vemos una diferencia significativa entre el 2009-2011 ( $p=0.03)$ mejorando su registro.

En el gráfico 1 se pude ver el porcentaje de registro de los cuidados de todo el periodo de estudio según el turno. Podemos observar que en todos los cuidados el mayor cumplimiento se registra en el turno de mañanas con una media global de $90,28 \%(D E 13,63)$ y el peor en el turno de noches con una media global de $83,69 \%$ (DE 10,46).

Gráfico 1: Cuidados registrados por turno en los protocolos del paciente con ventilación mecánica en el periodo 2009-2011.

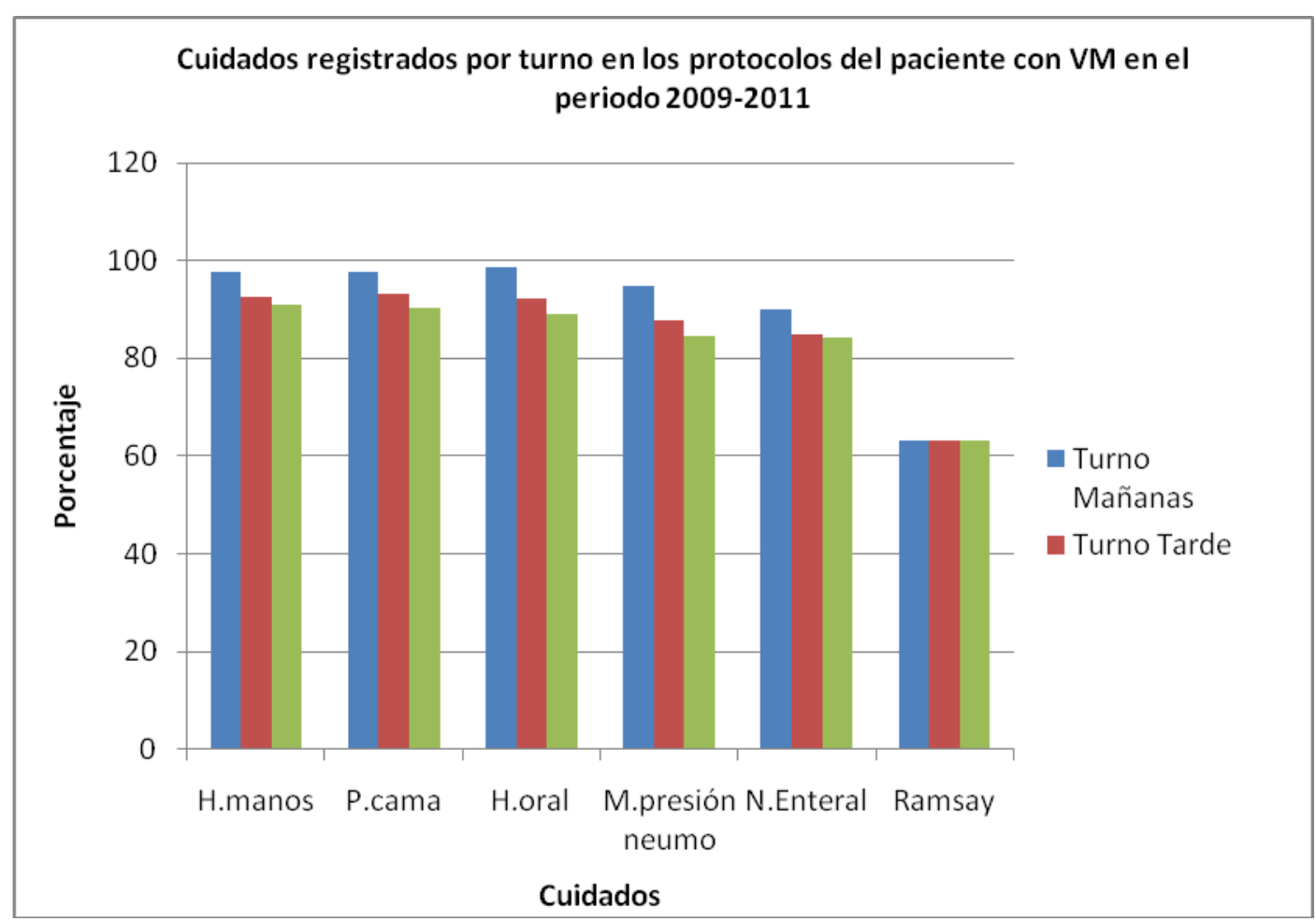

Durante todo el periodo del estudio se registraron 8 casos de NAVM; 3 en 2009, 4 en 2010 y 1 en 2011, donde se generaron respectivamente 656 días de VM en 2009, 586 días de VM en 2010 y 369 días de VM en 2011. El número de episodios de NAVM fue 4,57 en 2009, 6,83 en 2010 y 2,71 en 2011. Los microorganismos aislados más frecuentes fueron: 4 casos de Pseudomona aeruginosa, 2 casos de Candida albicans, 1 caso de Staphylococcus aureus, 1 caso de enterobacter aerogenes, 1 caso de Acinetobacter baumanni y 1 caso de Haemophilus influenzae. 


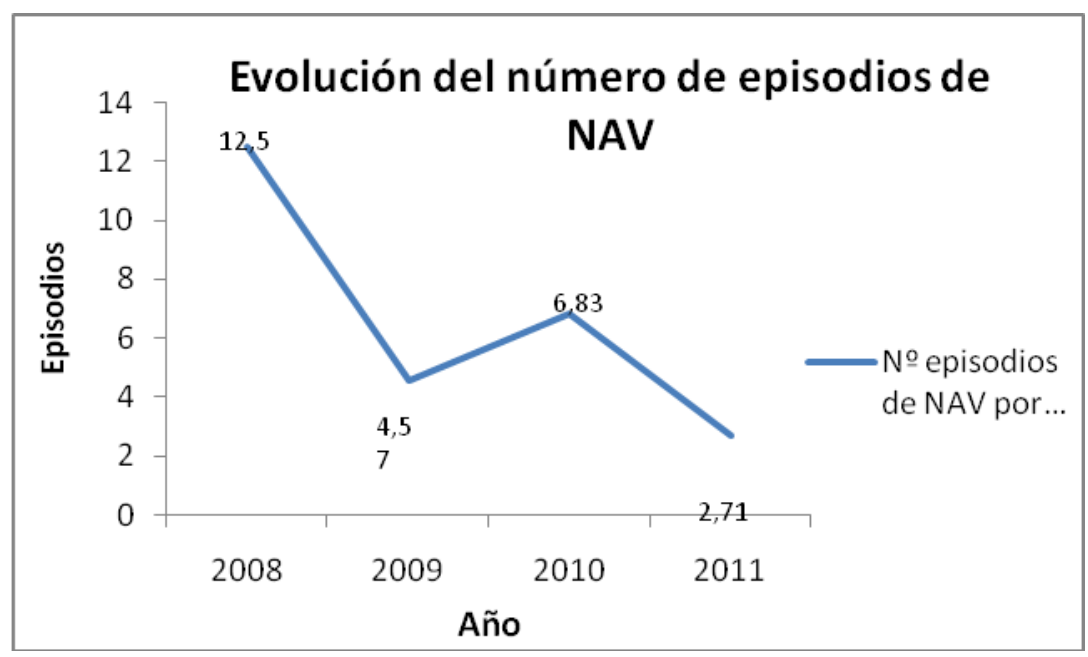

\section{DISCUSIÓN}

Respecto a las características de los pacientes podemos decir que son pacientes de riesgo porque la edad media es $>65$ años y la puntación de APACHE es $>16$, factores descritos en la bibliografía ${ }^{(4,5,11)}$ como de alto riesgo para desarrollar NAVM. Por otro lado en los estudios publicados por Dodek $\mathrm{P}^{(6)}$ y Elorza Mateos $\mathrm{J}^{(7)}$ se habla de que el riesgo de desarrollar NAVM aumenta de 6 a 21 veces si la ventilación mecánica es superior a 24 horas, siendo la media del estudio de 11,04 días por lo que el riesgo antes mencionado aún es mayor.

Con el análisis realizado se pude decir que el registro de todos los cuidados menos la valoración de la escala de sedación ha sido superior al $80 \%$, incluso en la mayoría de los casos ha superado el $90 \%$. A pesar de no existir una diferencia significativa en algunos cuidados, cabe destacar que este dato se acerca a valores más significativos si comparamos el año 2009 con 2011, por lo que podemos deducir que el registro mejora en 2011 con respecto al 2009. Este hecho seguramente se deba a una mayor integración del protocolo a la rutina diaria mediante la incorporación en las gráficas de enfermería de los cuidados que hay que realizar, ayudando a una planificación mejor y más sistematizada.

Sin embargo, cabe una mención especial, al mal porcentaje de registro de los cuidados en el turno noche en comparación con el resto de turnos, hecho que habría que analizar para poder corregirlo.

Con respecto al registro del cuidado de la cabecera de la cama (cuidado que menos se cumple según Rose $\left.L^{(18)}\right)$ no podemos considerarlo como una medida preventiva bien realizada ya que no se dispone de un instrumento de medida y son las enfermeras las que subjetivamente colocan al paciente en la posición de semisentado. Sí es verdad, que en las medidas de obligado cumplimiento del Protocolo de prevención de NAVM ${ }^{(8)}$ se habla solo de evitar la posición de decúbito supino $0^{\circ}$ y en el artículo escrito por Díaz LA ${ }^{(5)}$ se recomienda posición semisentada 
especialmente en pacientes que reciban nutrición enteral, no haciendo recomendación sobre los grados de elevación pero, a pesar de esto, habría que introducir un instrumento de medida para asegurar su fiabilidad.

El cuidado de comprobación de tolerancia de la nutrición enteral a través del volumen residual es el que mejores resultados ha obtenido aumentado su cumplimiento año a año y por tanto disminuyendo el riesgo de aspiración junto con la posición semisentada.

El control de la presión del globo de neumotaponamineto también tuvo un aumento progresivo en el registro. El cuidado se realiza como mínimo una vez por turno siguiendo las recomendaciones mínimas existentes ${ }^{(2,8,9)}$ pero a finales del año 2011 se hace una propuesta de mejora para el 2012 y la unidad dispondrá de dispositivos de medición continua de la presión que es la forma ideal de realizarlo según estos mismos estudios, por lo que este cuidado pasará a ser realizado en el $100 \%$ de los casos.

La escala Ramsay utilizada para la valoración de la sedación en los pacientes intubados fue la peor registrada (en torno al 60\%) y solo en un paciente constaba que se había realizado ventana de sedación. Hay que destacar, que en el 2011 el registro aumenta, seguramente debido a la formación proporcionada en marzo del mismo año a través de curso impulsado por el proyecto "Neumonía Zero".

En consonancia con esto, habría que plantearse si es necesario disponer de un protocolo específico de destete para mejorar este cuidado ya que es una medida importante como demuestra el Institute for Health Improvement (IHI) de Massachussets ${ }^{(1)}$ que identificó la interrupción diaria de la sedación junto con la valoración diaria de la posibilidad de destete del ventilador como una de las 4 intervenciones claves para disminuir la NAVM. Además este protocolo facilitaría el trabajo diario a las enfermeras, ya que según el artículo de Giménez Maroto AM $^{(1)}$ es uno de los cuidados más difíciles y que más tiempo requieren.

Respecto a la incidencia de NAVM, se puede ver en el gráfico 2 la enorme disminución que se produce en el 2009 con la puesta en marcha de los cuidados con respecto al año anterior. En el 2010 se produce una pequeña subida (6,83 episodios NAVM/1000 días VM) para volver a descender en 2011 (2,71 episodios NAVM/1000 días VM) con la entrada de la unidad en el proyecto "Neumonía Zero".

En el 2009 es la propia unidad la impulsora del funcionamiento de algunas medidas para prevenir la NAVM al constatar el elevado número de infecciones por esta causa y proporciona al personal una mínima formación, pero es en el 2011 cuando el personal recibe una formación más extensa y completa a través de la plataforma web ENVIN_HELICS, reforzando el paquete de medidas preventivas e introduciendo nuevos cuidados a ese paquete. 
Está sobradamente demostrado que la formación continuada del personal (5, 9,12-15) junto con el uso de paquetes medidas y la información acerca del cumplimiento de las medidas, mejora la seguridad de los paciente y previene las infecciones ${ }^{(27)}$.

Tras la puesta en marcha de esto, podemos observar que la tasa de NAVM es la más baja de la unidad y el mantenimiento del objetivo propuesto por el Proyecto "Neumonía Zero" de mantener el número de episodios de NAVM por debajo de $9{ }^{\left({ }^{8}\right)}$ se cumple. La coincidencia del gran descenso de la incidencia con el momento en que se empieza a implantar el protocolo con el paquete de medidas preventivas puede deberse al azar, pero como menciona la investigación de García Araguas ${ }^{(27)}$, los resultados coinciden con otras publicaciones que tras implementar programas plurifactoriales de prevención se consiguió disminuir la tasa de NAVM.

Como limitación del estudio encontramos que al ser un estudio retrospectivo la calidad del registro puede variar y nos podemos encontrar variables que han sido mal registradas, pero a pesar de esto, en nuestra investigación, se han podido evaluar todos los cuidados.

Por otro lado, para poder establecer una relación causa-efecto entre la disminución de la incidencia de NAVM y la implantación del protocolo sería necesaria una investigación prospectiva controlando de manera más exhaustiva las variables que pueden influir.

\section{CONCLUSIONES}

- Todos los cuidados registrados dentro del programa de prevención de NAVM han sido mayores del $80 \%$ menos la valoración de la escala de Ramsay por lo que sería necesario la elaboración e implementación de un protocolo dirigido a facilitar el destete. Por otro lado, se hace necesario la incorporación de instrumentos de medida para la medición de los grados de elevación de la cabecera de la cama.

- Los datos descritos muestran una disminución de la tasa de incidencia de NAVM tras la implantación del protocolo.

\section{Agradecimientos}

A todo el equipo de la unidad de cuidados intensivos del Hospital Obispo Polanco por la gran disponibilidad recibida para la realización del trabajo, a la dirección del mismo hospital por facilitarme el acceso a la documentación necesaria y a los profesores del máster por los conocimientos y seminarios recibidos y la alta disponibilidad para resolver todo tipo de dudas a pesar de la distancia 


\section{REFERENCIAS}

- (1) Giménez Maroto AM. Programa de valoración enfermera del destete y resultados de la ventilación mecánica. REDUCA (Enfermería, Fisioterapia y Podología). 2009;1(2): 316-31.

- (2) Marcos D, Ferrer I, Belkys Rodriguez L, Cruz de los Santos H, Pereira Valdés E. Guía de práctica clínica para el tratamiento de la neumonía asociada al ventilador. Medisur 2009;7(1):supl

- (3) Yifan Xue. ¿Cuál es la mejor evidencia disponible sobre prevención de la neumonía asociada a ventilador (NAV)?. Instituto Joanna Briggs. Agosto 2010

- (4) Fernandez J, Ochoa M, Grajeda P, Guzman E, Lugo M, Gonzalez J. Guia neumonias intrahospitalarias.[Internet]. [acceso 10 Enero 2010]. Disponible: http://www.diresacusco.gob.pe/inteligencia/epidemiologia/guias/GUIA\%20NEU MONIAS\%2OINTRAHOSPITALARIAS.pdf

- (5) Díaz LA, Llauradó M, Rello J, Restrepo MI. Prevención no farmacológica de la neumonía asociada a ventilación mecánica. Archivos de Bronconeumología. 2010;46(4):188-95

- (6) Dodek P, Keenan S, Cook D, Heyland D, Jacka M, Hand L, et al. EvidenceBased Clinical Practice Guideline for the Prevention of Ventilator-Associated Pneumonia. Am J Crit Care 2004;141:305-13

- (7) Elorza Mateos J, Ania González N, Ágreda Sádaba M, Del Barrio Linares M, Margall Coscojuela M, Asiain Erro M. Valoración de los cuidados de enfermería en la prevención de la neumonía asociada a ventilación mecánica. Enfermería Intensiva. 2011;22(1):22-30

(8) Protocolo de prevención de las neumonías relacionadas con ventilación mecánica en las UCl españolas. $1^{\text {a }}$ edición, 2011. Publicado por el Ministerio de Sanidad, Política Social e Igualdad de España. La Sociedad española de Medicina intensiva, crítica y unidades coronarias (SEMICYUC) y la Sociedad española de Enfermería intensiva y unidades coronarias (SEEIUC). [Internet]. [acceso 7 Enero 2012]. Disponible: http://www.semicyuc.org/sites/default/files/protocolo nzero.pdf

- (9) Garcia R, Arias S, Vázquez M, Jam R, Sanchez M, De la Cal MA, Gordo F, Álvarez J, Añon M, Palomar M, Lorente L, Alvarez Lerma F. Informe de los expertos de la Sociedad Española de Medicina Intensiva, Crítica y Unidades Coronarias y de la Sociedad española de Enfermeria de Medicina Intensiva y Unidades Coronarias. [Internet] [acceso 7 Enero 2012]. Disponible: http://www.semicyuc.org/sites/default/files/informe-revision-expertos.pdf

- (10) E. Diaz, L. Lorente, J. Valles, J. Rello. Mechanical ventilation associated pneumonia. Med Intensiva 2010 Jun-Julio 2010;34(5):318-24.

- (11) Raurell Torredà M. Impacto de los cuidados de enfermería en la incidencia de neumonía asociada a la ventilación mecánica invasiva. Enferm Intensiva 2011;22(1):31-8.

- (12) Coffin SE, Klompas M, Classen D, Arias KM, Podgorny K, Anderson DJ, et al. Strategies to prevent ventilator-associated pneumonia in acute care hospitals. Infect Control Hosp Epidemiol 2008 Oct;29 Suppl 1:S31-40. 
- (13) Prevention of ventilator associated pneumonia. In: Betsy Lehman Center for Patient Safety and Medical Error Reduction, JSI Research and Training Institute, Inc. Prevention and control of healthcare-associated infections in Massachusetts. Part 1: final recommendations of the Expert Panel. Boston (MA): Massachusetts Department of Public Health; 2008 Jan 31. p. 56-60.

- (14) Taylor N, van Saene HK, Abella A, Silvestri L, Vucic M, Peric M. Selective digestive decontamination. Why don't we apply the evidence in the clinical practice?. Med Intensiva 2007 Apr;31(3):136-45.

- (15) Arlene F. Tolentino-DelosReyes, Susan D. Ruppert and Shyang-Yun Pamela K. Shiao. Evidence-Based Practice: Use of the Ventilator Bundle to Prevent Ventilator-Associated Pneumonia. Am J Crit Care 2007;16:20-7.

- (16)Lorente L, Blot S, Rello J. Evidence on measures for the prevention of ventilator-associated pneumonia. Eur Respir J 2007 Dec;30(6):1193-1207.

- (17) Klompas M. Prevention of ventilator-associated pneumonia. Expert Rev Anti Infect Ther 2010 Jul;8(7):791-800

- (18) Rose L, Baldwin I, Crawford T, Parke R. Semirecumbent positioning in ventilator-dependent patients: a multicenter, observational study. Am. J. Crit. Care. 2010;19(6):100-8

- (19) Miquel-Roig C, Pico-Segura P, Huertas-Linero C, Pastor-Martinez M. Nursing care related to the prevention of ventilator-associated pneumonia: a systematic review. Enferm Clinica 2006 Sep;16(5):244-52

- (20) Sole ML, Penoyer DA, Bennett M, Bertrand J, Talbert S. Oropharyngeal secretion volume in intubated patients: the importance of oral suctioning. Am. J. Crit. Care. 2011 Nov;20(6):141-5.

- (21) Peter JV, Chacko B, Moran JL. Comparison of closed endotracheal suction versus open endotracheal suction in the development of ventilator-associated pneumonia in intensive care patients: an evaluation using meta-analytic techniques. Indian J Med Sci 2007 Apr;61(4):201-11.

- (22) Siempos II, Vardakas KZ, Falagas ME. Closed tracheal suction systems for prevention of ventilator-associated pneumonia. $\mathrm{Br} J$ Anaesth 2008 Mar;100(3):299-306.

- (23) Subirana M, Solà I, Benito S editor. Sistemas de aspiración traqueal cerrados versus sistemas de aspiración traqueal abiertos para pacientes adultos con ventilación mecánica. $2^{\text {a }}$ ed.: revisión Cochrane; 2008

- (24) Lacherade JC, Auburtin M, Cerf Ch, van de Louw A, Soufir L, Rebufat Y, et al. Impact of humidification systems on ventilator-associated pneumonia. Am J Respir Crit Care Med. 2005;172:1276-82.

- (25) Han J, Liu Y. Effect of ventilator circuit changes on ventilator-associated pneumonia: a systematic review and meta-analysis. Respir Care 2010 Apr;55(4):467-74

- (26) Chan EY, Ruest A, Meade MO, Cook DJ. Oral decontamination for prevention of pneumonia in mechanically ventilated adults: systematic review and meta-analysis. BMJ 2007 Apr 28;334(7599):889

- (27) García Araguas T, Irigoyen Aristorena I, Zazpe Oyarzun C, Baztán Madoz B, Barado Hugalde J. Evaluación de un programa de prevención de neumonía 
asociado a ventilación mecánica: resultados al año. Enferm Intensiva 2012;23(1):4-10.

\section{ANEXOS:}

Anexo 1: Escala Acute Physiology Chronic Health Evaluation o APACHE II.

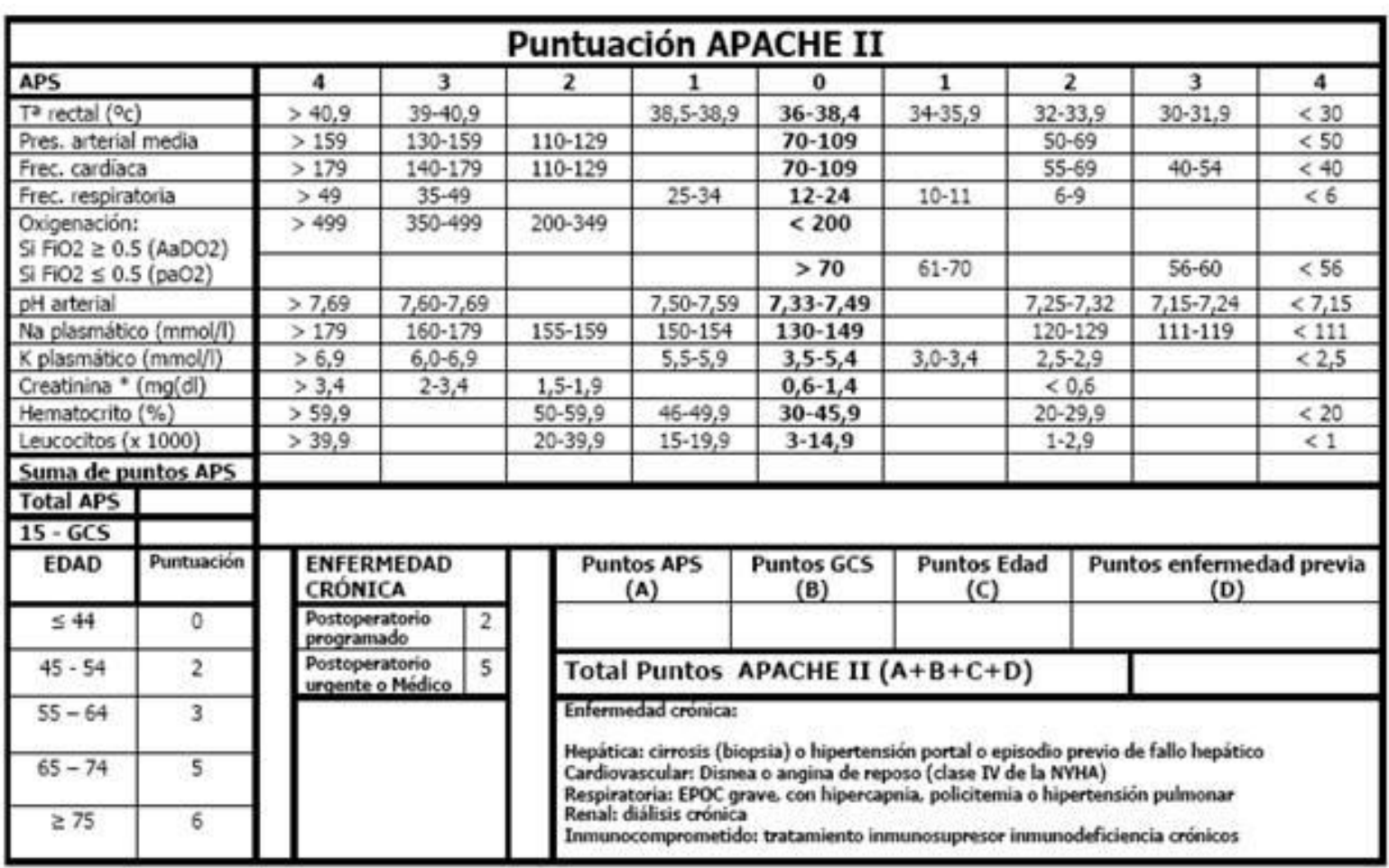

\section{Anexo 2:_Recomendaciones de sedación en paciente con Ventilación mecánica: Escala Ramsay}

Nivel 1: Despierto; ansioso y agitado o inquieto o ambos

Nivel 2: Despierto; colaborador, orientado y tranquilo.

Nivel 3: Despierto; responde a órdenes verbales

Nivel 4: Dormido; responde con viveza a toque frontal o estímulos auditivos fuertes.

Nivel 5: Dormido; respuesta tardía a toque frontal o estímulos auditivos fuertes.

Nivel 6: No responde a toque frontal o estímulos auditivos fuertes. 


\section{Anexo 3: Hojas de registro de los protocolos de la unidad.}

A. Evaluación del paciente con cuidados respiratorios en VM con o sin humificación activa.

PACIENTE:

MES:

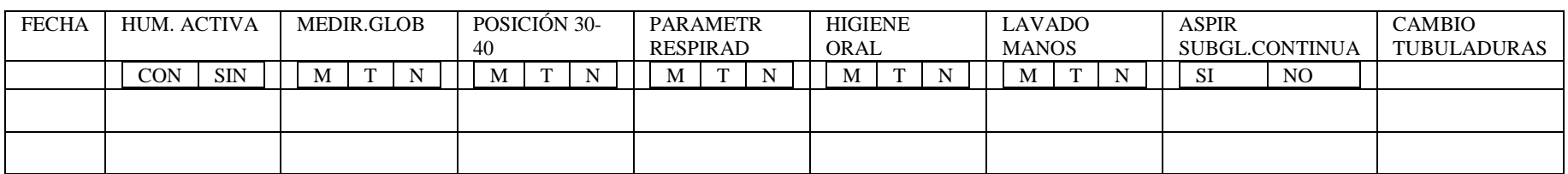

B. Evaluación del paciente con nutrición enteral y parenteral

PACIENTE:

\begin{tabular}{|c|c|c|c|c|c|c|c|c|}
\hline \multicolumn{5}{|c|}{ NUTRICION ENTERAL } & \multicolumn{4}{|c|}{ NUTRICION PARENTERAL } \\
\hline FECHA & RESIDUOS & PROBLEMAS & $\begin{array}{l}\text { CAMBIO } \\
\text { SISTEMA } \\
\text { C/24H }\end{array}$ & $\begin{array}{l}\text { LAVADO } \\
\text { SONDA }\end{array}$ & $\begin{array}{l}\text { CONEXIÓN } \\
\text { PROTEGIDA }\end{array}$ & $\overline{\text { GLUCEMIA }}$ & $\begin{array}{l}\text { VIA } \\
\text { UNICA }\end{array}$ & $\begin{array}{l}\text { CAMBIO } \\
\text { SISTEM } \\
\text { C/24H }\end{array}$ \\
\hline & \begin{tabular}{l|l}
$\mathrm{M}$ & $\mathrm{T}$ \\
\end{tabular} & & & \begin{tabular}{l|l|}
$\mathrm{M}$ & $\mathrm{T}$ \\
\end{tabular} & & & & \\
\hline
\end{tabular}

C. Evaluación del paciente con sedación

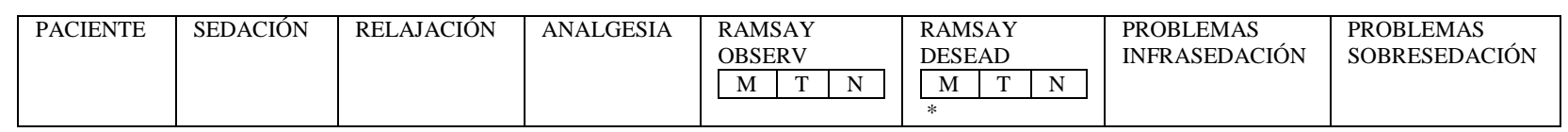

*EL ramsay deseado se empieza a registrar con la entrada al proyecto Neumonia Zero (marzo 2011)

Recibido: 30 de octubre 2013; Aceptado: 7 de febrero 2014 